\title{
The G305 Star-forming Region. I. Newly Classified Hot Stars*
}

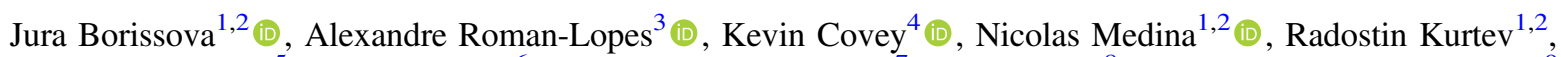 \\ Carlos Roman-Zuniga ${ }^{5}$ (D), M. A. Kuhn ${ }^{6}$ (D), Carlos Contreras Peña ${ }^{7}$, Philip Lucas ${ }^{8}$ (D), Sebastian Ramirez Alegria ${ }^{9}$ (D), \\ Dante Minniti $^{2,10,11}$ (D), Marina Kounkel ${ }^{12}$ (D), Guy Stringfellow ${ }^{13}$ (D), Rodolfo H. Barbá ${ }^{3}$, and Genaro Suárez ${ }^{14}$ (D) \\ ${ }^{1}$ Instituto de Física y Astronomía, Universidad de Valparaíso, Av. Gran Bretaña 1111, Playa Ancha, Casilla 5030, Chile; jura.borissova@uv.cl \\ ${ }^{2}$ Millennium Institute of Astrophysics (MAS), Santiago, Chile \\ ${ }^{3}$ Departamento de Física y Astronomía, Universidad de La Serena, La Serena, Chile \\ ${ }^{4}$ Department of Physics and Astronomy, Western Washington University, Bellingham, WA 98225, USA \\ ${ }^{5}$ Instituto de Astronomía en Ensenada UNAM, Ensenada BC 22860, Mexico \\ ${ }^{6}$ Department of Astronomy, California Institute of Technology, Pasadena, CA 91125, USA \\ ${ }^{7}$ Department of Physics and Astronomy, University of Exeter, Stocker Road, Exeter, Devon EX4 4SB, UK \\ ${ }^{8}$ Centre for Astrophysics, University of Hertfordshire, College Lane, Hatfield, AL10 9AB, UK \\ ${ }^{9}$ Centro de Astronomía (CITEVA), Universidad de Antofagasta, Avenida Angamos 601, Antofagasta, Chile \\ ${ }^{10}$ Departamento de Física, Facultad de Ciencias Exactas, Universidad Andés Bello, Av. Fernandez Concha 700, Las Condes, Santiago, Chile \\ ${ }^{11}$ Vatican Observatory, V00120 Vatican City State, Italy \\ ${ }^{12}$ Department of Physics and Astronomy, Western Washington University, 516 High Street, Bellingham, WA 98225, USA \\ ${ }^{13}$ Center for Astrophysics and Space Astronomy, Department of Astrophysical and Planetary Sciences, University of Colorado, 389 UCB Boulder Colorado, USA \\ ${ }^{14}$ Instituto de Astronomía, Universidad Nacional Autónoma de México, Unidad Académica en Ensenada, Ensenada BC 22860, México \\ Received 2019 January 24; revised 2019 May 31; accepted 2019 June 3; published 2019 July 8
}

\begin{abstract}
The relatively nearby star-forming complex G305 is one of the most luminous H II regions in the Galaxy, and it contains several sites and epochs of star formation. Using a combination of near-infrared photometry from "Vista Variables in Via Lactea" ESO Large Public Survey, SDSS-IV APOGEE-2 spectra, and Gaia DR2 photometry and astrometry, we report on 29 OB type, Wolf-Rayet, and emission-line stars, 18 of which are newly classified. Most of these hot stars belongs to the main sequence, although some stars of class I are also proposed. The mean radial velocity is $\mathrm{RV}=-41.8 \mathrm{~km} \mathrm{~s}^{-1}$. The average spectroscopic distance is $3.2 \pm 1.6 \mathrm{kpc}$, while the Gaia DR2 average distance is $3.7 \pm 1.8 \mathrm{kpc}$. Eight objects show light-curve variations with amplitudes greater than $0.5 \mathrm{mag}$ in the $K_{S}$ band.
\end{abstract}

Key words: Galaxy: stellar content - infrared: stars - stars: massive - stars: Wolf-Rayet - stars: variables: general

\section{Introduction}

Located within the Scutum-Crux arm of the Milky Way ( $l=305^{\circ} .506, b=00^{\circ} .085$ ), the G305 star-forming complex is both relatively nearby (a heliocentric distance of $3.8 \pm 0.6 \mathrm{kpc}$ ) and one of the most luminous H II regions in the Galaxy (Davies et al. 2012; Urquhart et al. 2014). The aggregate is centered on and driven by the two young $\left(1.5_{-0.5}^{+1.5} \mathrm{Myr}\right.$ and $\left.3.0_{-1.0}^{+3.0} \mathrm{Myr}\right)$, massive $\left(8000 \pm 1500 M_{\odot}\right.$ and $\left.3000 \pm 800 M_{\odot}\right)$ star clusters Danks 1 and Danks 2 (Chené et al. 2012; Davies et al. 2012), which appear to be associated with an extended population of distributed massive stars. At larger projected distances from Danks 1 and Danks 2 we have identified four young stellar groups via the "VVV-Vista variables in the Via Lactea" ESO Public Survey (Minniti et al. 2010; Saito et al. 2012), namely VVV CL 021; CL 022; CL 023 and CL 024 (Borissova et al. 2011; Chené et al. 2012). Furthermore, evidence of ongoing star formation is found around the periphery of the large wind-blown cavity formed by Danks 1 and Danks 2, with numerous H II regions, infrared hotspots, masers and dense molecular structures (Clark \& Porter 2004; Hindson et al. 2010, 2012, 2013; Faimali et al. 2012; Urquhart et al. 2014). Twenty-three star clusters, 11 Wolf-Rayet (WR) stars (Mauerhan et al. 2011; Chené et al. 2012; Davies et al. 2012), and 17 mid to late $O$ supergiants (Davies et al. 2012) are cataloged so far. The region contains several distinct sites and epochs of star formation, which permits the study of massive

* Based on Vista Variables in the Vía Láctea (VVV); SDSS-IV APOGEE-2 and Gaia DR2 data. star formation and evolution, as well as their impact on the surrounding environment. The morphology of the region and the distribution of young stars suggest that interaction between the evolved massive stars and the remaining natal molecular material is taking place, resulting in an ongoing star formation activity (Figure 1). Coupled with its relatively close distance, this makes G305 an excellent laboratory for a multiwavelength study of triggered, sequential star formation (Clark et al. 2011). Much of the work has already been carried out in understanding the earliest stages of star formation in this region, with radio and submillimeter observations providing a census of the deeply embedded protostars and proto-clusters (e.g., Hindson et al. 2010, 2012, 2013; Faimali et al. 2012; Urquhart et al. 2014). A variability study of high-amplitude YSOs has been conducted by Contreras Peña et al. (2017).

However, there presently have been no homogeneous, multiwavelength, and time-domain studies of the resolved stellar population of the whole region. Therefore, we have initiated a program to characterize this population, employing infrared, optical, and X-ray photometry, $K_{S}$-band light-curve analysis, moderately high-resolution spectroscopy, and Gaia DR2 proper motion and distances.

In this paper, the first of our series, we analyze the most massive stellar cohort of G 305, namely WR, O, and B stars. New near-infrared spectral observations are performed with the APOGEE-2 spectrograph. Twenty-nine O, B, and WR stars are analyzed, 18 of them are newly classified. Their positions on the color-magnitude diagrams and variability status are discussed. In the subsequent papers, we will report on the 

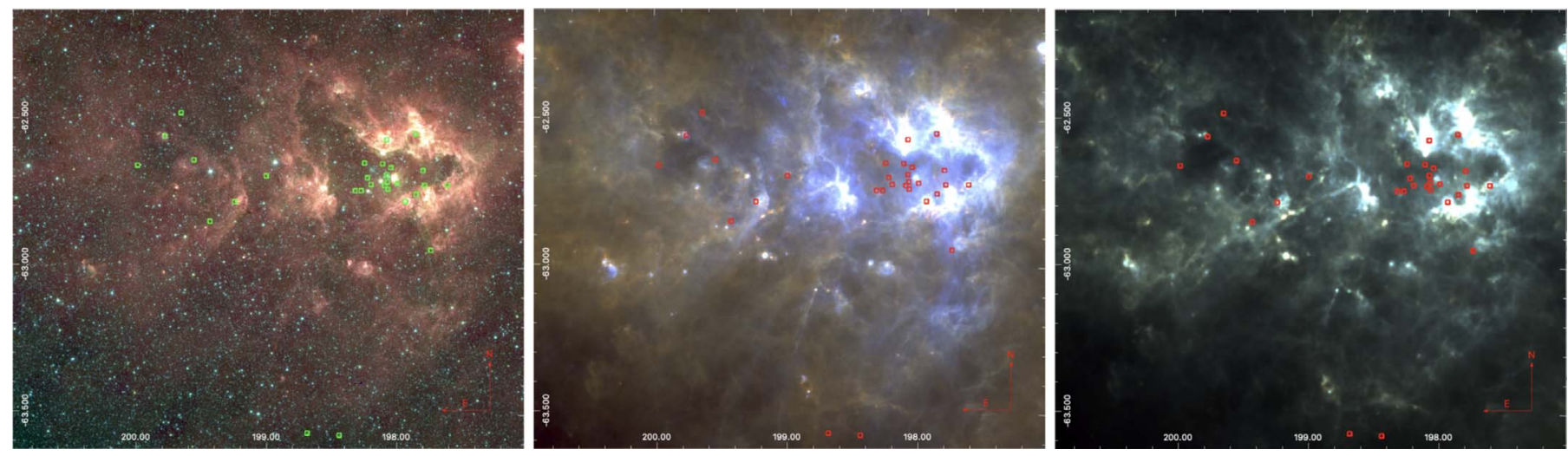

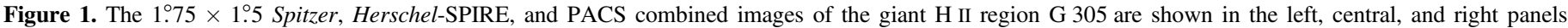

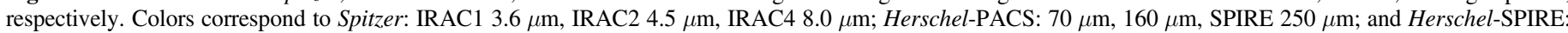
$250 \mu \mathrm{m}, 350 \mu \mathrm{m}, 500 \mu \mathrm{m}$. The projected position of the OB stars (circles) reported in this study is shown.

young stellar object (YSO) population and star clusters in the region.

\section{Target Selection, Observations, and Data Reduction}

Apache Point Observatory Galaxy Evolution Experiment (APOGEE-2) is a second-generation near-infrared spectrograph, mounted on the $2.5 \mathrm{~m}$ du Pont Telescope at Las Campanas Observatory in Chile. The observed spectral range is $1.51-1.70 \mu \mathrm{m}$, with resolution $R=22,500$. Approximately 300 stars can be observed per plate: typically assigning about 250 fibers for science targets, 35 for sky, and 15 for telluric stars, with the fiber collision limit of about $70^{\prime \prime}$.

Our science target selection was based on Robitaille et al. (2008) and Faimali et al. (2012) catalogs of YSOs; our own variability searches based on VVV $K_{S}$-band multi-epoch survey (see Contreras Peña et al. 2017 and Medina et al. 2018 for search methods) and some probable cluster members from Chené et al. (2012) and Borissova et al. (2016) studies. We have also used the Chandra Source Catalog (version 1.1; Evans et al. 2010), which provides 289 X-ray sources from a single 120 ks Chandra observation, of which 113 have matches to the Two Micron All Sky Survey (2 MASS; Skrutskie et al. 2006). With the APOGEE-2 magnitude limit of $H<13$ mag we have included $56 \mathrm{X}$-ray selected stars in targeting. Furthermore, we have selected 12 known WR and OB stars from the SIMBAD ${ }^{15}$ database, in order to test the performance of the instrument, as our observations were conducted shortly after the first light of the APOGEE Southern hemisphere installation.

The observations were made on 2017 April 14 (under external CNTAC program No. CN2016B90), with $1 \mathrm{hr}$ exposure times. The corresponding $\mathrm{S} / \mathrm{N}$ ratio of the observed stars is given in Table 3. The data were processed by the APOGEE Stellar Parameter and Chemical Abundances Pipeline (ASPCAP; Nidever et al. 2015), which includes basic data reduction, combinations of spectra from multiple visits, and measurement of radial velocities, stellar parameters, and elemental abundances. ${ }^{16}$ Additionally, the spectra were reprocessed using routines and methods similar to those used by Roman-Lopes et al. (2018), which include a rectification of individual spectra by applying the IRAF task continuum through the use of a SPLINE 3 function. Bad pixels were carefully

\footnotetext{
15 http://simbad.u-strasbg.fr/simbad/

16 For more details see http://www.sdss.org/dr14/algorithms/.
}

removed by hand using the splot task in IRAF. The equivalent widths (EWs) were measured from the continuum-normalized spectra using the same task. The radial velocities were obtained by the IRAF task fxcor, using two radial velocities standards: HD1544 and BD57247. Despite the high spectral resolution of the APOGEE-2 spectrograph, the radial-velocity errors are relatively large (between 5 and $10 \mathrm{~km} \mathrm{~s}^{-1}$ ), due to the small number of lines (4-5) available for early-type stars. Two stars from our sample (2M13122855-6241438 and 2M13183535-6229284) have radial-velocity measurements in the literature (Chené et al. 2012; Sota et al. 2014), and these are in reasonable agreement with our own measurements (less than $10 \%$ difference).

\section{Spectral Classification of the Individual Objects}

In total, $29 \mathrm{WR}, \mathrm{O}, \mathrm{B}$, and emission-line stars were identified from our APOGEE-2 observations based on comparison of their spectral features with known stars of similar spectral type. They were then classified using empirical calibration described in Roman-Lopes et al. (2018). In general, this classification was calibrated based on the EWs of the APOGEE-2 observed spectra to measure the spectral type of the stars using 92 known OB stars. The hydrogen lines of the Brackett series $\lambda 16811$ (11 -4), $\lambda 16113$ (13-4), and $\lambda 15705$ (15-4) (hereafter Br11, $\mathrm{Br} 13$, and $\mathrm{Br} 15$, respectively), and two He II lines, $\lambda 15723$ (7 -13) and $\lambda 16923(7-12)$ (hereafter He II(7-13) and He II (7-12), respectively) are calibrated by linear regression (see Equations (1)-(6) of Roman-Lopes et al. 2018). The EWs of the stars observed in this work are given in Table 1. The errors are calculated taking into account the uncertainties of the continuum normalization and the uncertainties of the individual spectral line fitting.

We report on five WR stars (Figure 2). As has been previously stated, four of them are known WR stars specifically selected to test the performance of the new instrument, and one is newly classified. Following the main goals of the APOGEE survey, the instrument and the corresponding pipeline are designed to observe and analyze red giant stars. Thus, it was necessary to investigate the quality of data products for hot stars, which contain, in the best case, only 4-5 spectral lines in the observed spectral range. The notes of the individual objects are as follows:

The object 2MASS J13120905-6243267 is a WN8-9 star discovered by Mauerhan et al. (2011; (object [MVM2011b] 
Table 1

Equivalent Widths (EWs) of the Program Stars

\begin{tabular}{lccc}
\hline \hline Name & EW (Br13) & EW(Br11) & EW(He II (7-12)) \\
& $\AA$ & $\AA$ & $\AA$ \\
\hline 2M13103971-6243315 & $4.74 \pm 0.43$ & $3.25 \pm 0.29$ & $\ldots$ \\
2M13110621-6256547 & $4.15 \pm 0.33$ & $2.20 \pm 0.18$ & $\ldots$ \\
2M13112057-6243378 & $6.60 \pm 0.46$ & $4.50 \pm 0.32$ & $\ldots$ \\
2M13112385-6240410 & $0.74 \pm 0.04$ & $3.16 \pm 0.19$ & $\ldots$ \\
2M13113936-6233138 & $0.72 \pm 0.07$ & $1.08 \pm 0.11$ & $\ldots$ \\
2M13115442-6247078 & $1.18 \pm 0.11$ & $2.33 \pm 0.21$ & $0.03 \pm 0.01$ \\
2M13122653-6243107 & $0.52 \pm 0.03$ & $0.36 \pm 0.02$ & $0.61 \pm 0.03$ \\
2M13122965-6234341 & $1.24 \pm 0.12$ & $0.81 \pm 0.08$ & $\ldots$ \\
2M13123199-6243583 & $0.81 \pm 0.06$ & $1.56 \pm 0.11$ & $0.54 \pm 0.04$ \\
2M13123629-6239301 & $1.33 \pm 0.12$ & $1.31 \pm 0.12$ & $0.94 \pm 0.07$ \\
2M13125612-6243503 & $0.92 \pm 0.06$ & $1.84 \pm 0.13$ & $1.66 \pm 0.12$ \\
2M13130268-6242256 & $0.44 \pm 0.04$ & $3.13 \pm 0.25$ & $0.35 \pm 0.02$ \\
2M13130841-6239275 & $0.64 \pm 0.06$ & $2.46 \pm 0.25$ & $0.38 \pm 0.03$ \\
2M13131324-6245056 & $1.34 \pm 0.12$ & $1.57 \pm 0.14$ & $1.51 \pm 0.11$ \\
2M13132400-6245070 & $0.71 \pm 0.07$ & $2.50 \pm 0.25$ & $0.66 \pm 0.05$ \\
2M13134553-6335116 & $0.44 \pm 0.04$ & $0.55 \pm 0.05$ & $\ldots$ \\
2M13144440-6334518 & $0.66 \pm 0.07$ & $0.28 \pm 0.03$ & $\ldots$ \\
2M13165955-6247413 & $6.65 \pm 0.47$ & $4.04 \pm 0.28$ & $\ldots$ \\
2M13174367-6251410 & $1.88 \pm 0.11$ & $2.49 \pm 0.15$ & $\ldots$ \\
2M13181288-6239067 & $1.86 \pm 0.09$ & $5.42 \pm 0.27$ & $\ldots$ \\
2M13183535-6229284 & $0.89 \pm 0.08$ & $0.69 \pm 0.06$ & $0.25 \pm 0.02$ \\
2M13190396-6234102 & $1.50 \pm 0.13$ & $1.30 \pm 0.12$ & $\ldots$ \\
2M13195292-6240096 & $3.29 \pm 0.30$ & $2.88 \pm 0.26$ & $\ldots$ \\
\hline & & & \\
\hline & & &
\end{tabular}

MDM3). We can only compare the EWs of the He II(7-12) line. Mauerhan et al. (2011; see their Table 3) reported $\mathrm{EW}_{\mathrm{He} \mathrm{II(7-12)}}=2.0 \pm 0.2 \AA$, while we measured $\mathrm{EW}_{\text {He II(7-12) }}=2.2 \pm 0.2 \AA$, which is in good agreement within the errors. Although Mauerhan et al. (2011) do not provide any numerical value for the hydrogen lines, the visual inspection of both spectra shows that they have similar behavior, and that the $\mathrm{He} \mathrm{II}(7-12)$ line is stronger than the Br11 one. We measure $\mathrm{EW}_{\mathrm{Br} 11}=1.09 \AA$.

2MASS J13122130-6240125 was discovered by Shara et al. (2009), and it was subsequently observed by Mauerhan et al. (2011) ([MVM2011b] MDM4). No EW measurements are reported by Mauerhan et al. (2011) and their $H$-band spectrum has $R=800$, thus it is difficult to perform a visual comparison. Our spectrum shows all hydrogen lines (Br16, Br15, Br14, $\mathrm{Br} 13, \mathrm{Br} 12$, and $\mathrm{Br} 11)$ in emission, while $\mathrm{He} \mathrm{II}(7-12)$ is in absorption.

2MASS J13122546-6244417 was observed by Mauerhan et al. (2011) ([MVM2011b] MDM5). Both He II lines (7-13 and 7-12) are in emission, together with all hydrogen lines. We measure smaller than Mauerhan et al. (2011) $\mathrm{EW}_{\mathrm{He} \mathrm{II}(7-12)}=1.34 \pm 0.14$. But in general, comparing visually, both spectra have similar behavior.

Thus, we can conclude that the instrument is useful for observations of WR stars. Nevertheless, for these three WR stars we adopted the spectral types as given in the abovementioned papers, as they are based on a wider spectral range.

The star 2MASS J13122855-6241438 was discovered by Mauerhan et al. (2011) ([MVM2011b] MDM8) and observed by Chené et al. (2012; their Obj 31 , only $K$-band spectrum). This star is a confirmed member of the Danks 1 cluster (Mauerhan et al. 2011; Chené et al. 2012). As pointed out by Mauerhan et al. (2011), the Brackett lines (Br11, Br12, Br13, etc.) are in emission, but the diagnostic He II lines show complex behavior. In the $H$ band (at $16923 \AA$ ) a weak P Cygni profile is detected, while in the $K$ band the line is in absorption (at $21860 \AA$ ). Thus, the authors tentatively classify MDM8 as a WN9h star, but do not exclude an $\mathrm{O}$ emission-line supergiant type, possibly in transition to a late WN star. They pointed out

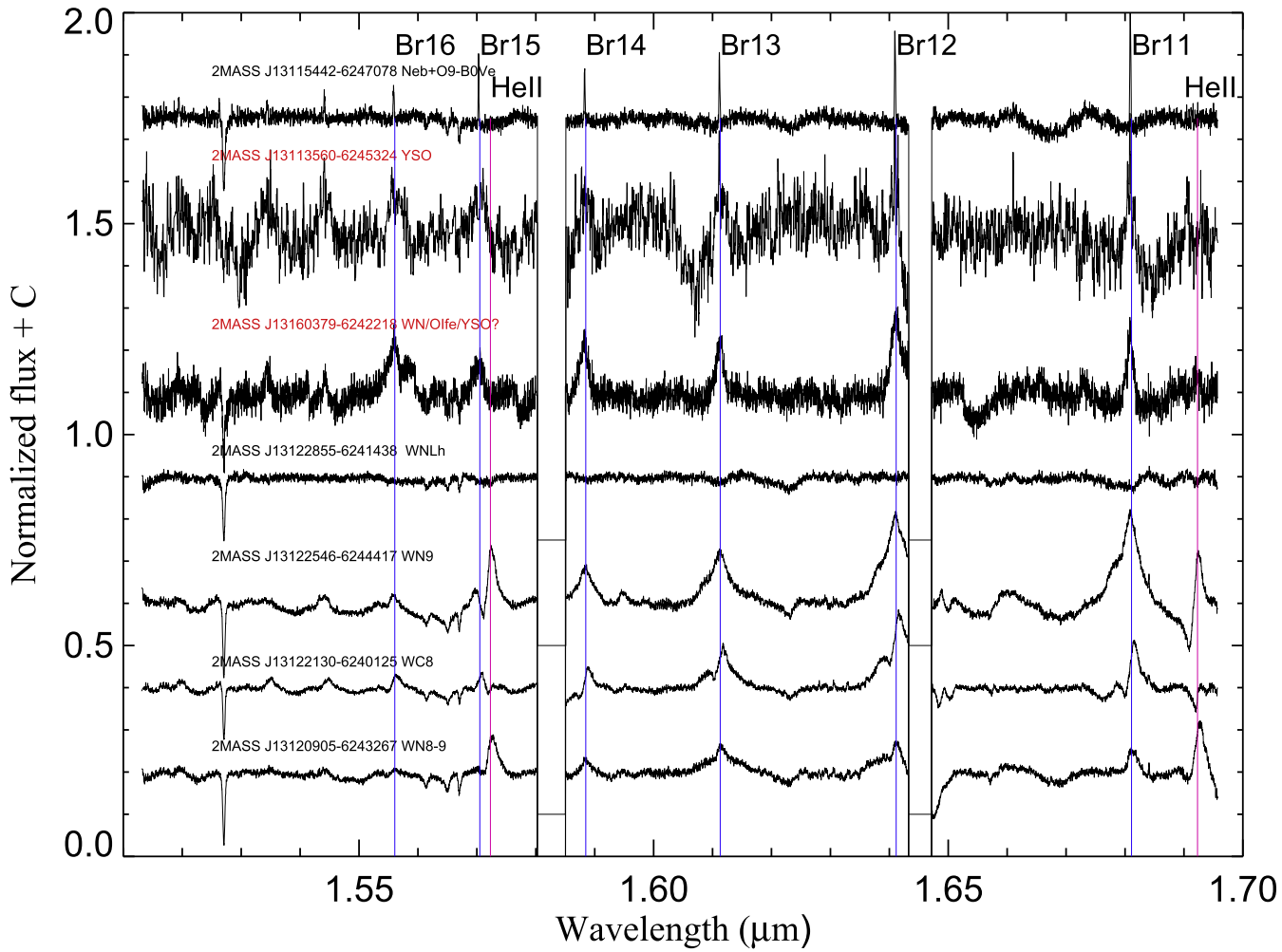

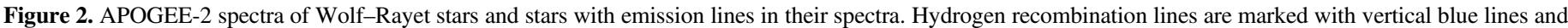
helium lines are marked with vertical red ones. All stars are labeled. The red indicates newly proposed WRs and YSOs. 


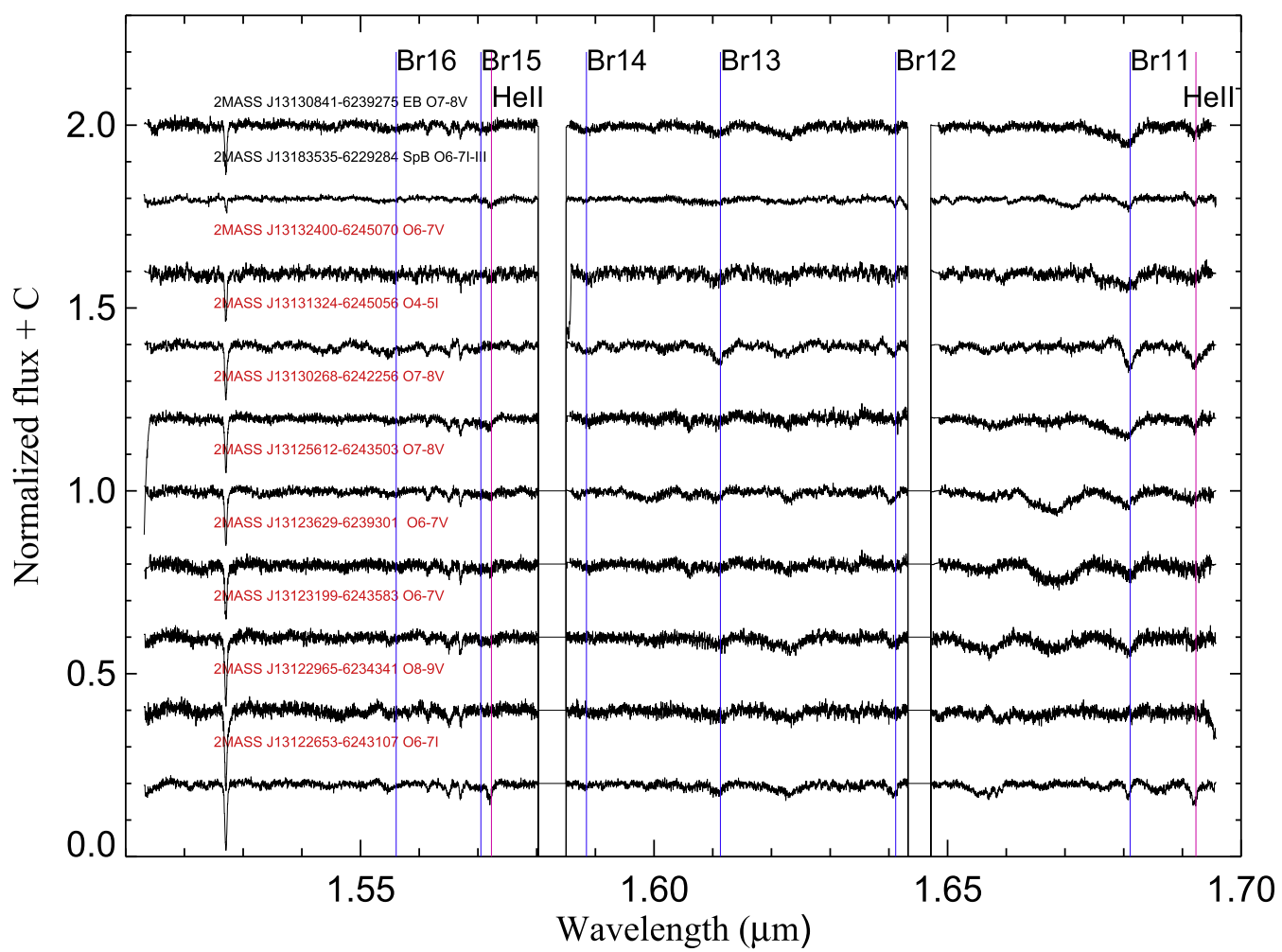

Figure 3. APOGEE-2 spectra of O stars. The symbols are the same as in Figure 2.

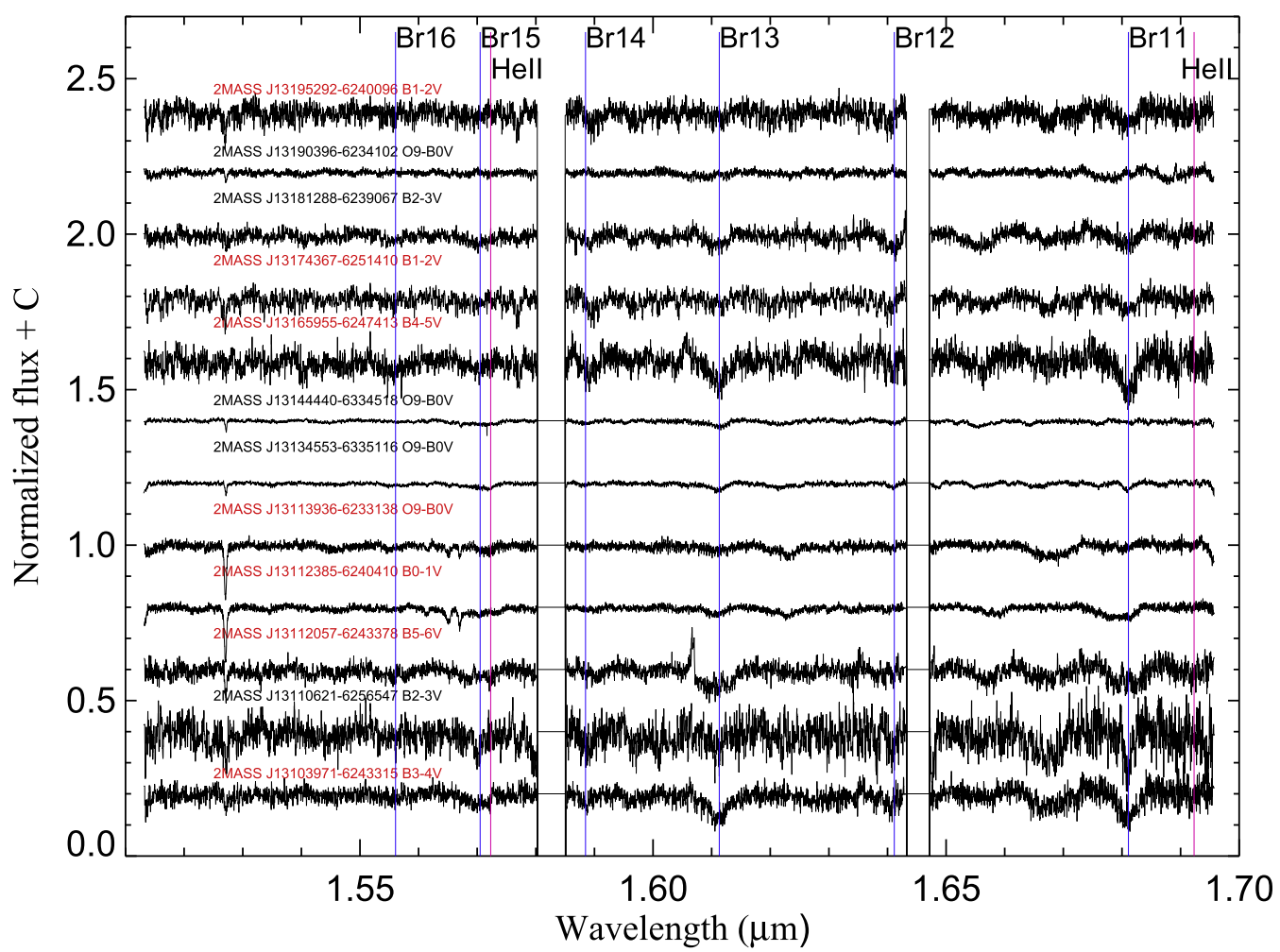

Figure 4. APOGEE-2 spectra of B stars. The symbols are the same as in Figure 2.

that higher-S/N data with increased spectral resolution is necessary for a firmer classification. Our APOGEE spectrum $(R=22,500$ at $\mathrm{S} / \mathrm{N}=150)$ shows all of the Brackett lines in absorption within the observed spectral range. The He II line (at $16923 \AA$ ) is also in absorption, with
$\mathrm{EW}_{\mathrm{He} \mathrm{II}(7-12)}=0.38 \pm 0.05$, indicating an O7-8I spectral type using the calibration from Roman-Lopes et al. (2018). It seems that our observations support the hypothesis of a late-type $\mathrm{O}$ supergiant that is possibly in transition. 

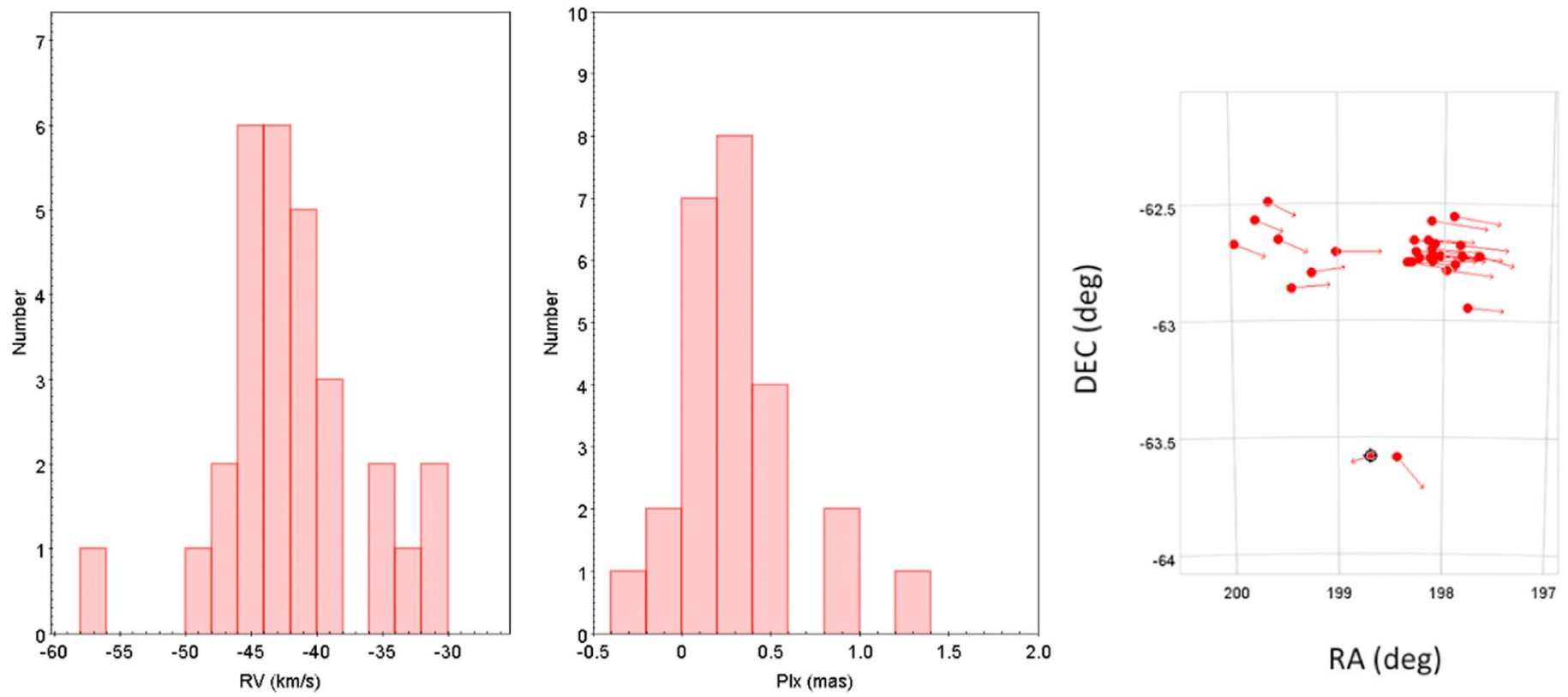

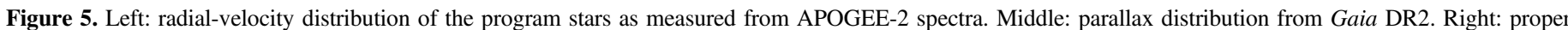
motion distribution from Gaia DR2. The proper motion vectors are scaled by a factor of 2 for visibility.
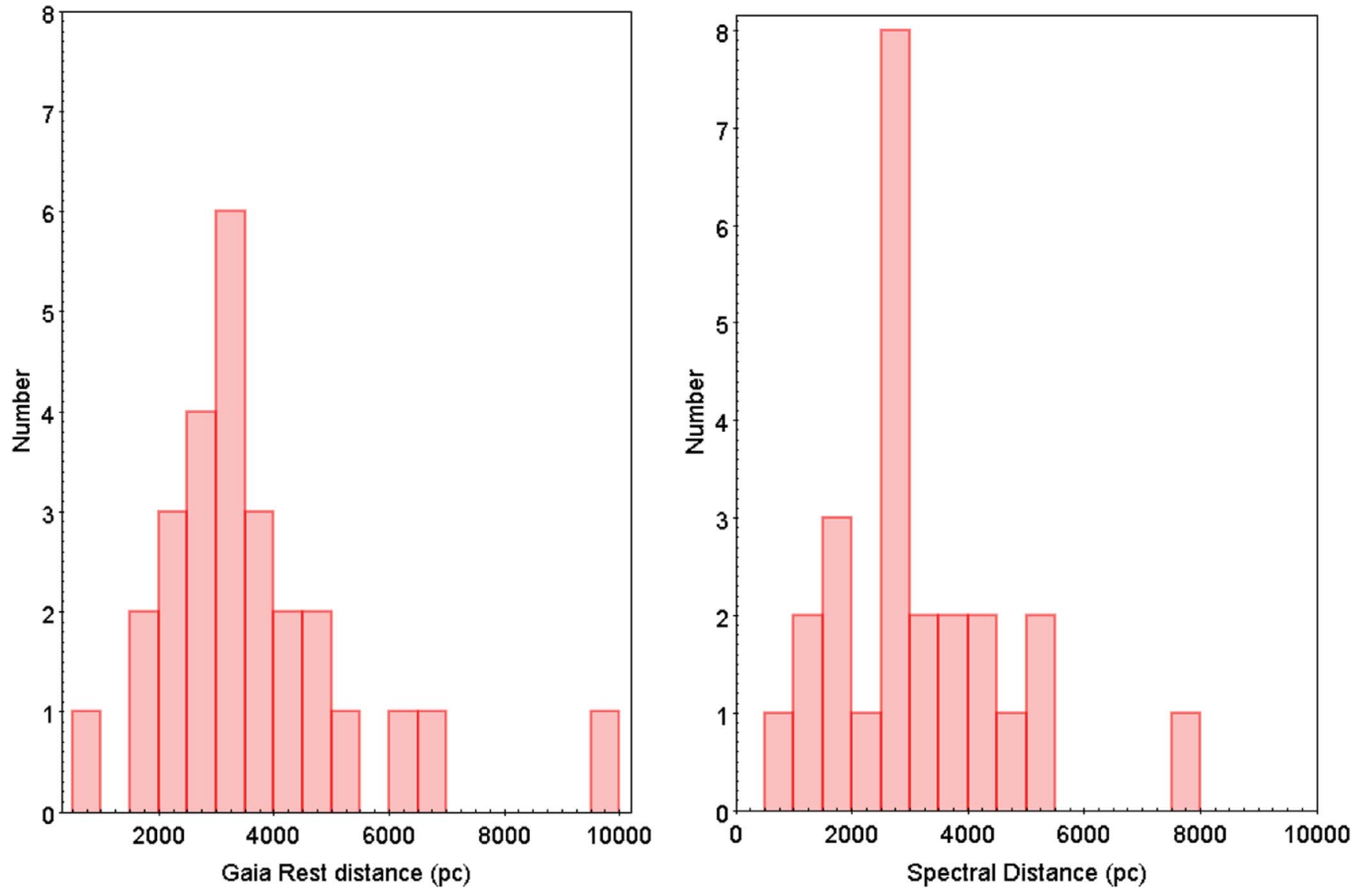

Figure 6. Astrometric (left) and spectroscopic (right) distance determination.

The source 2MASS J13160379-6242218 is the only new WR star candidate. This star was classified as a YSO by Robitaille et al. (2008) and by Marton et al. (2016) on the basis of GLIMPSE and WISE photometry. Although the $\mathrm{S} / \mathrm{N}=31$ of our spectrum is relatively low, the emission of Brackett lines is clearly visible. The He II(7-12) line also shows some weak emission. The EWs are $\mathrm{EW}_{\mathrm{Br} 13}=2.23 \pm 0.20$, $\mathrm{EW}_{\mathrm{Br} 13}=2.90 \pm 0.14, \quad$ and $\mathrm{EW}_{\mathrm{He} \mathrm{II}(7-12)}=2.14 \pm 0.15$. Thus, we are proposing a OIf*/WN spectral type. Taking into account, however, that we have only a narrow $H$-band spectral 

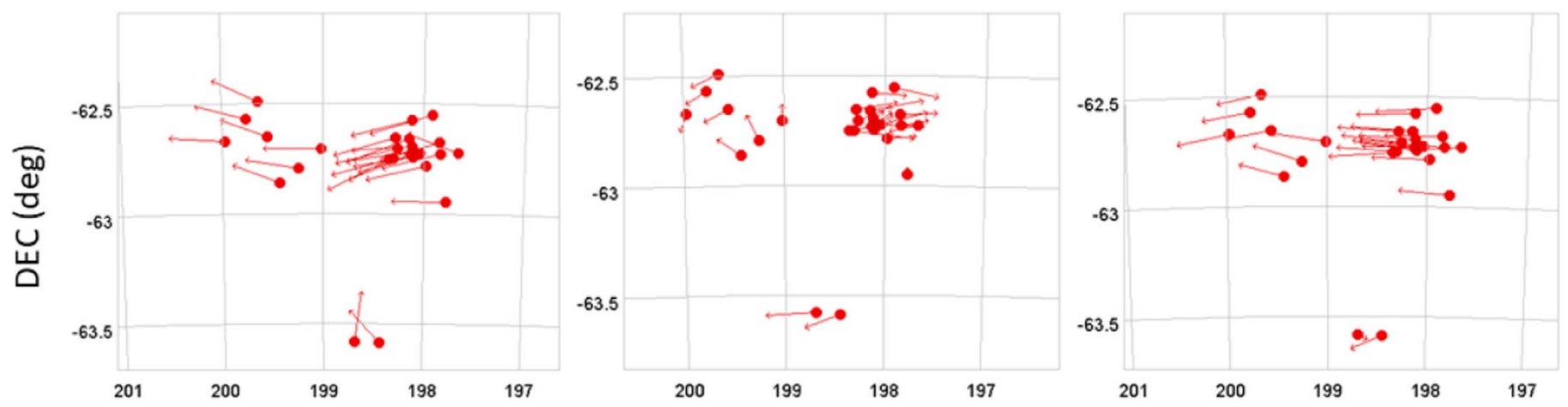

RA (deg)

Figure 7. From left to right: the $U V, V W$, and $U W$ space velocities (arrows), scaled by a factor of 2 for visibility.
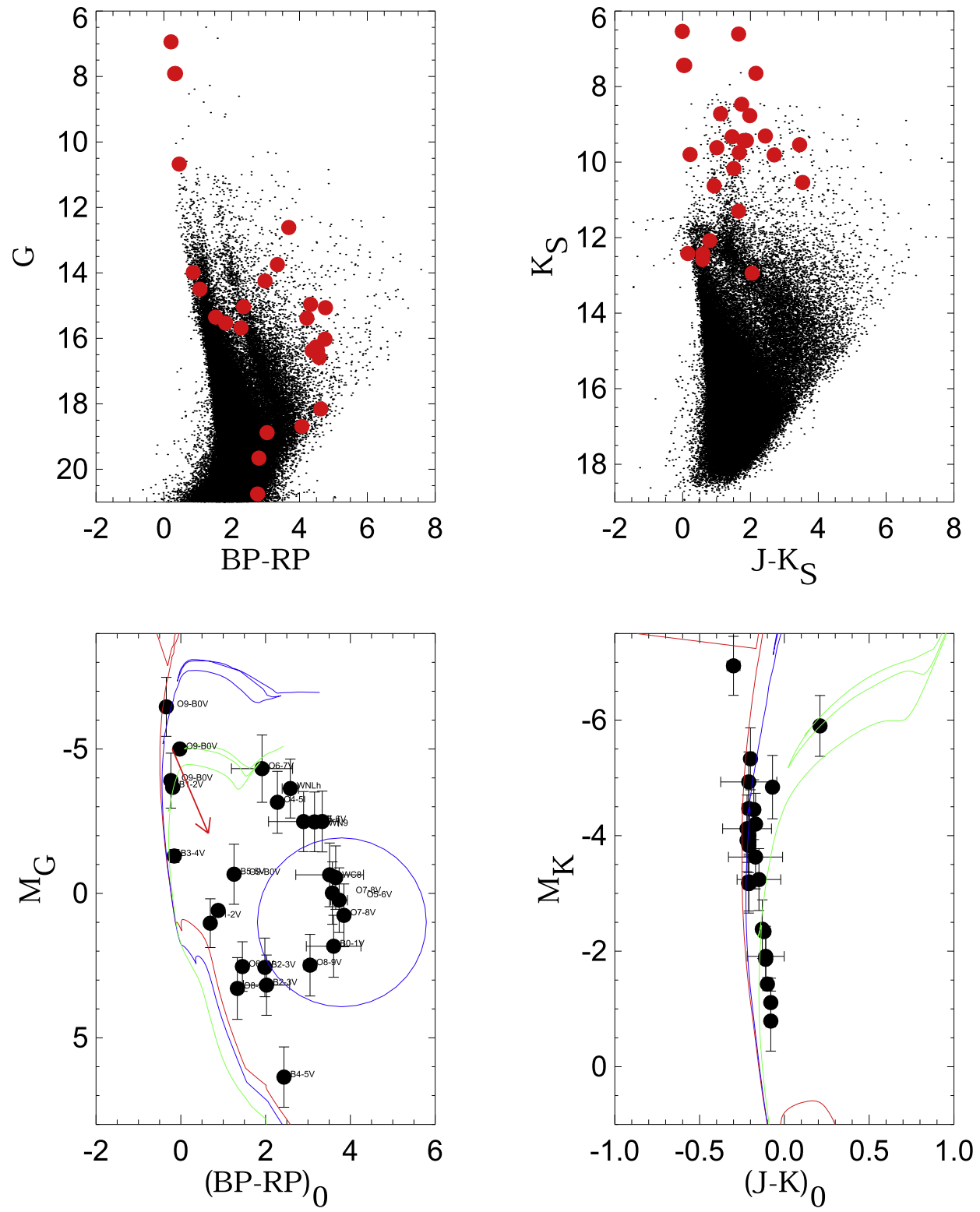

Figure 8. Upper panel: the BP-RP vs. $G$ mag Gaia DR2 and $K_{S}$ vs. $\left(J-K_{S}\right)$ color-magnitude diagrams, with program stars overplotted (red circles). Lower panel: the absolute color-magnitude diagrams, with the spectral types of the stars labeled. The red arrow is the mean reddening vector. The solid lines are 5 (red), 10 (blue), and 50 (green) Myr Padova isochrones.

range, this classification should be taken as preliminary, and the 

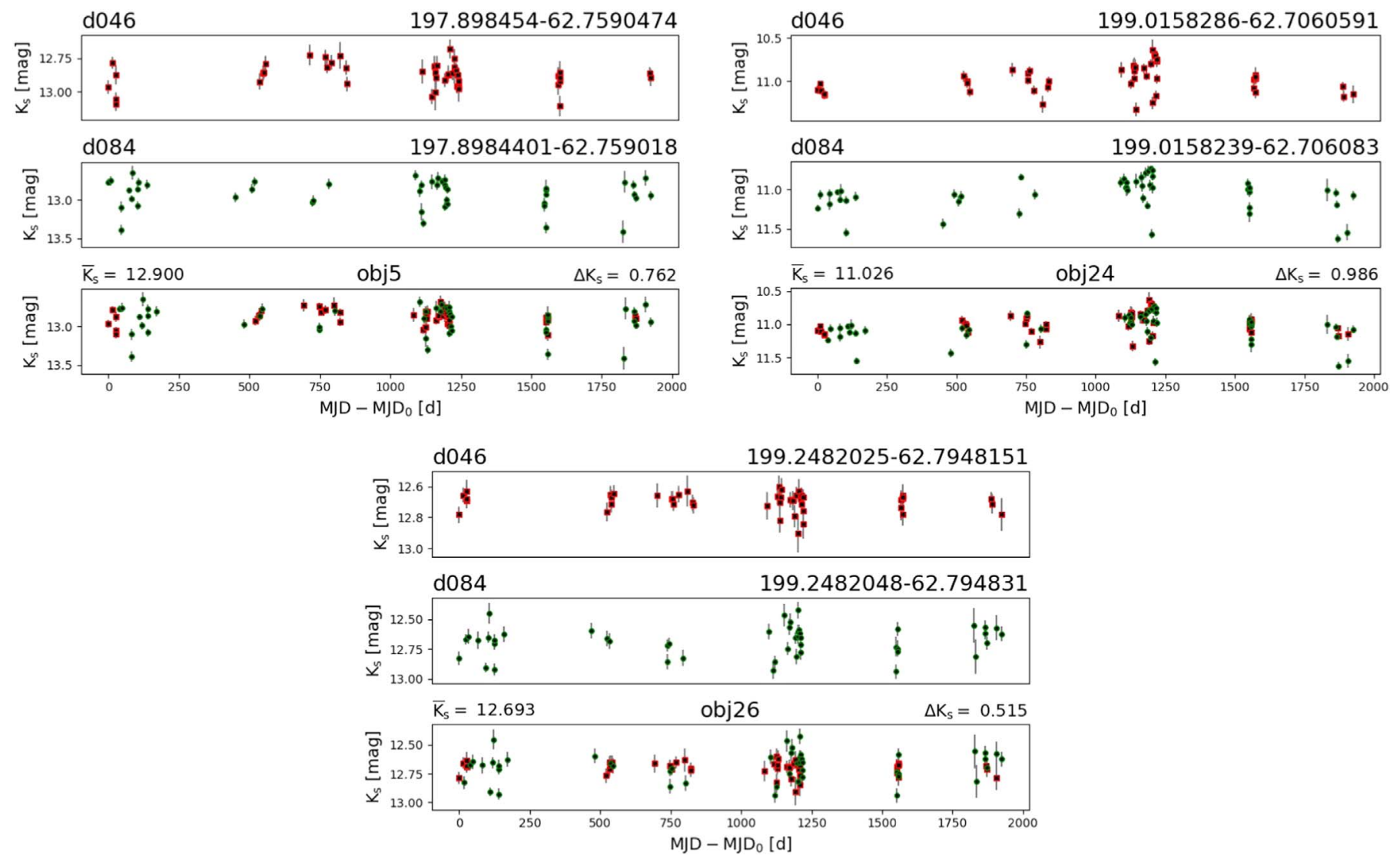

Figure 9. From left to right: 2MASS J13113560-6245324 (Obj 5), 2MASS J13160379-6242218 (Obj 24), and 2MASS J13165955-6247413 (Obj 26) overlapping light curves from the VVV survey.

star could be also a YSO with accretion.

Although the $\mathrm{S} / \mathrm{N}$ of the spectrum of the star 2MASS $\mathrm{J} 13113560-6245324$ is low $(\mathrm{S} / \mathrm{N}=7)$, some weak emission of hydrogen lines can be detected. The object is classified as Class 0/I YSO by Faimali et al. (2012), and we confirm this status.

The spectrum of the star 2MASS J13115442-6247078 shows some typical nebulosity in hydrogen lines in emission. This is expected, as it is a member of the young star cluster [DBS2003] 130, which is still embedded in the H II region [HTU2013] G305.270-0.007. The star is observed in the $K$ band by Borissova et al. (2016; object [BRA2016] DBS130 Obj2), and the spectrum shows hydrogen lines and HeI $(2.06 \mu \mathrm{m})$ in emission. It is classified as B0Ve. Using the empirical calibration of Roman-Lopes et al. (2018) we obtained a O9V spectral type, which is in agreement within 2 spectral sub-types. Thus, we adopted the spectral type of O9-B0Ve.

Nine O stars were also classified (Figure 3). The star 2MASS $\mathrm{J} 13183535-6229284$ is a well known spectroscopic binary. Sota et al. (2014) assigned this star a O8III((f)) type, while the Roman-Lopes et al. (2018) empirical classification gives a O67 I-III spectral type. This is a reasonable agreement, but the classification of Sota et al. (2014) is adopted, as it is based on optical spectra that allow a more robust classification.

2MASS J13130841-6239275 is an eclipsing binary, reported by Kourniotis et al. (2015), who found components of O6.5-O7 spectral types. We cannot separate the components, but found a similar spectral type for the combined spectrum, $\mathrm{O} 7-8 \mathrm{~V}$. The star is member of the Danks 2 cluster, according to Kourniotis et al.
The remaining stars are newly classified O-type mainsequence and supergiant stars.

Twelve dwarf B stars are reported here (Figure 4); seven of them are newly classified. 2MASS J13190396-6234102 is a member of the Stock 16 cluster (Vázquez et al. 2005), and it is classified as B2V star, while our classification derives the slightly younger spectral type O9-B0V.

The stars 2MASS J13181288-6239067 and 2MASS J13110621-6256547 are YSO candidates from Robitaille et al. (2008). There are no emission lines in our spectra, and we classify the stars as B2-3V.

The sources 2MASS J13144440-6334518 and 2MASS J13134553-6335116 are either double or higher-order multiple stars, according to SIMBAD. We cannot resolve their components in our spectra, which leads to uncertain classification.

The spectrum of the star 2MASS J13112057-6243378 shows broad, double hydrogen lines, indicating a possible binary system.

\section{Fundamental Parameters of the Stars from APOGEE-2 and Gaia DR2}

The Gaia spacecraft was launched in 2013 December (Gaia Collaboration et al. 2016) to measure positions, parallaxes, proper motions, and photometry for over $10^{9}$ sources, as well as to obtain spectra and measure radial velocities for millions of stars. Its recent Data Release 2 (Gaia DR2) has covered the initial 22 months of the mission (Gaia Collaboration et al. 2018a). As pointed out by Gaia Collaboration et al. (2018b), it is expected that the members of the clusters and star-forming 

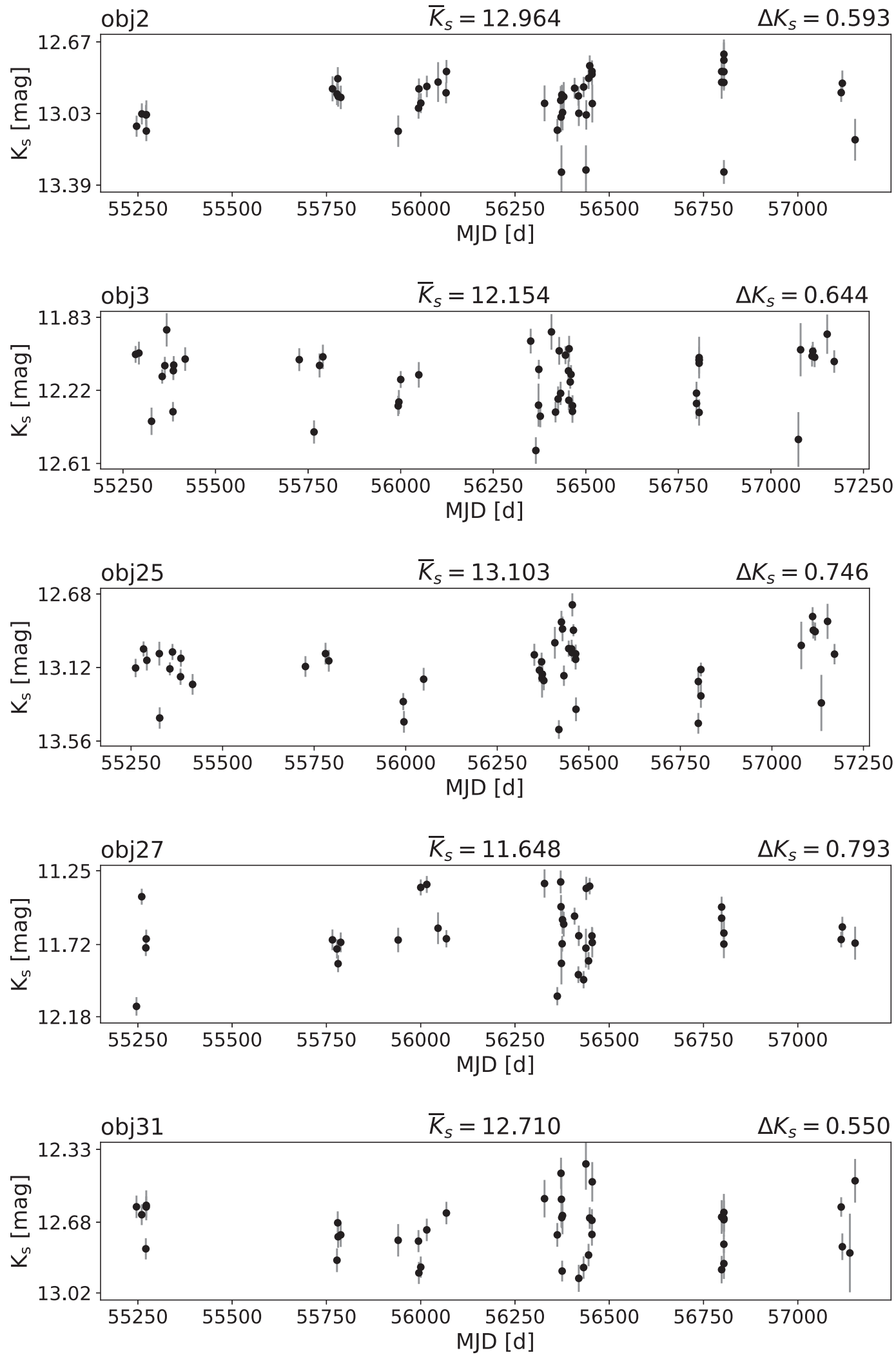

Figure 10. Light curves of the variable sources 2M13110621-6256547 (Obj 2); 2M13112057-6243378 (Obj 3); 2M13165540-6242318 (Obj 25); 2M13174367-6251410; (Obj 27); and 2M13195292-6240096 (Obj 31).

regions span a small range of distances, and their members have similar kinematics, different from the bulk motion of the field stars in the same region. The cross identification of our sample with Gaia DR2, with matching radius 1", shows that only two stars (2M13122130-6240125 and 2M13125612-6243503) have no measured parallaxes and proper motions. 

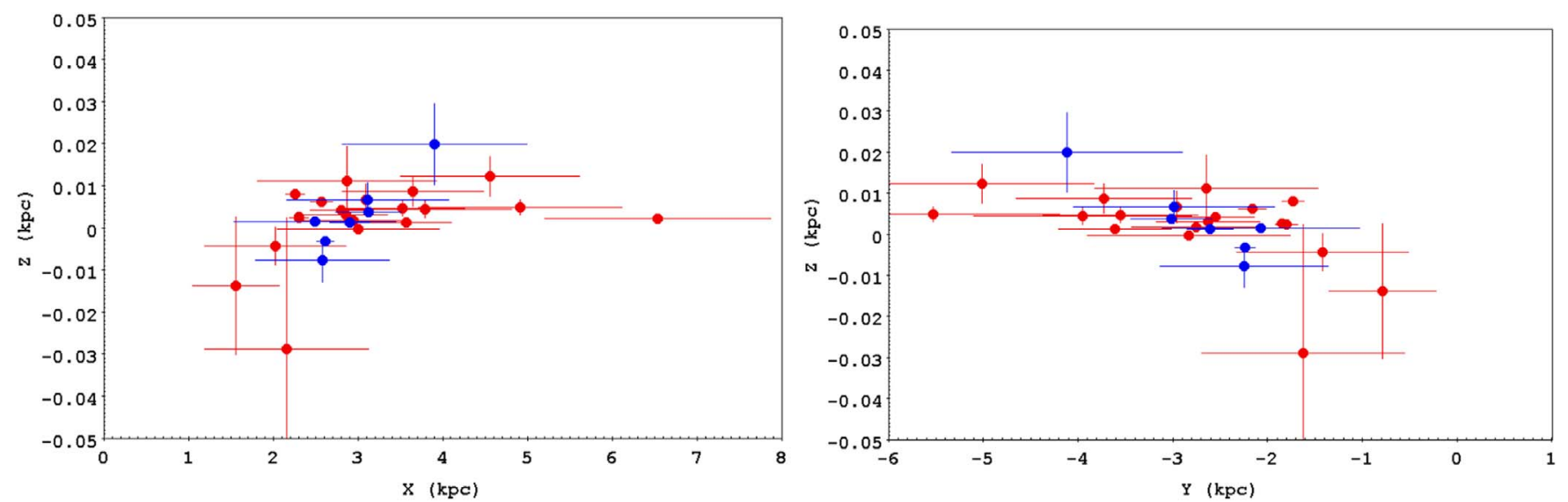

Figure 11. Projections on the $X Z$ and $Y Z$ planes of positions of $O B$ stars. The Sun lies at $(Y ; X)=(0 ; 0)$. The blue circles show variable stars.

We expect that stars formed in the G305 region have relatively similar velocities. Figure 5 shows the distribution of radial velocities of the observed stars as measured from APOGEE-2 spectra. Indeed, the radial velocities of most stars are distributed between -50 and $-39 \mathrm{~km} \mathrm{~s}^{-1}$, with the peak of the best-fit Gaussian at $\mathrm{RV}=-41.8 \mathrm{~km} \mathrm{~s}^{-1}$, with a standard deviation of $\sigma=5.4 \mathrm{~km} \mathrm{~s}^{-1}$. This value is in good agreement with Chené et al. (2012) mean radial velocities of the star clusters Danks 1 and Danks $2\left(-42 \pm 1\right.$ and $-44 \pm 1 \mathrm{~km} \mathrm{~s}^{-1}$, respectively). Figure 5 also shows the distribution of parallaxes and proper motions of the observed stars as given by Gaia DR2. The observed median parallax is between 0.2 and 0.4 mas, consistent with the spectroscopically obtained distance value (see Table 3). In the right panel of Figure 5 the proper motion vectors are plotted, outlying three groups with similar proper motions. The proper motions of the stars 2M1314440-6334518 and 2M13134553-6335116, both classified as O9-B0V type stars, differ significantly, and they may be field stars.

The distance to the stars was calculated by two methods: the spectroscopic parallaxes based on the assigned spectral type (for more details on the method see Borissova et al. 2014, 2016), and Gaia DR2 distance measurements of Bailer-Jones et al. (2018). The average spectroscopic distance is $3.23 \pm 1.58 \mathrm{kpc}$, while the Gaia average value gives $3.65 \pm 1.76 \mathrm{kpc}$, comparable within the errors. The histogram distribution is shown in Figure 6.

To study the three-dimensional motion of stars, we calculate the $U V W$-velocities using the radial velocity, the proper motion components, and the Gaia DR2 distance using IDL program gal-uvw.pro (W. Landsman 2000). The output velocities are corrected for the solar motion $(U, V, W)_{\odot}=(-8.5,13.38,6.49)($ Coşkunoğlu et al. 2011) to the local standard of rest. Figure 7 shows the $U V, V W$, and $U V$ vectors, confirming the groups visible in the proper motion diagram.

Figure 8 shows observed (not corrected for the extinction) optical Gaia's DR2 $\left(G_{\mathrm{BP}}-G_{\mathrm{RP}}\right)$ versus $G$ and near-infrared $\left(J-K_{S}\right)$ versus $K_{S}$ color-magnitude diagrams of the region, with the program stars overplotted (red circles). Most stars appear to belong to the main-sequence population. The absolute magnitudes and colors are calculated using the individually calculated distances and reddening, both in Gaia and VVV systems. The individual extinction and distance was estimated using the spectral classifications of the objects and the intrinsic colors and luminosities cited by Martins \& Plez (2006) for O type stars, and by Straižys \& Lazauskaitė (2009) for the other spectral types. The uncertainties are calculated by quadratically adding the photometric errors and the uncertainty in the spectral classification (e.g., 2 subtypes). Gaia DR2 provided extinction measurements for some of the targets. The stars without reddening estimates are dereddened by values of the closest neighbor star. To compare, we plotted the 5, 10, and $50 \mathrm{Myr}$ Padova model isochrones (Bressan et al. 2012). Although the errors are large, due to the distance and reddening uncertainties, the stars appear to follow the corresponding sequences closely. The primary exception to this is a group around $M_{G}=1$ and $(\mathrm{BP}-\mathrm{RP})_{0}=4$ (enclosed in the large blue circle in Figure 8), which need to be analyzed further.

\section{Variability}

The Vista Variables in the Vía Láctea (VVV) survey provides dozens of epochs of $K_{s}$-band measurements over a period of more than $5 \mathrm{yr}$. The Medina et al. (2018) automated tool for identifying variable sources was run on the VVV tiles d046 and d084, where our targets are projected. Around 50 epochs are available for these tiles, but a fraction of the stars are saturated in some images, so they have been removed from the light-curve analysis.

The VVV has been designed with a small overlap between tiles. In Figure 9 we show the light curves of 2MASS J13113560-6245324 (Obj 5), 2MASS J13160379-6242218 (Obj 24), and 2MASS J13165955-6247413 (Obj 26) projected in both tiles, as well as their composed light curves (Figure 9), in order to show their behavior. Figure 10 shows the rest of the light curves, namely for 2MASS J13110621-6256547 (Obj 2), 2MASS J13112057-6243378 (Obj 3), 2MASS J13165540-6242318 (Obj 25), 2MASS J13174367-6251410 (Obj 27), and 2MASS J13195292-6240096 (Obj 31). Some periodicity can be outlined. For example, 2MASSJ13113560-6245324 (Obj 5) shows some long-period behavior $\left(>2000^{d}\right)$, with a shorter period of $5.16^{d}$ superimposed. The 2MASS J13160379-6242218 (Obj 24) shows some periodicity around $8.14^{d}$, and could be an eclipsing binary. These periods, however, should be taken as preliminary.

In total, there are eight objects that show light-curve variations with amplitude greater than $0.5 \mathrm{mag}$ in $K_{S}$. All of them, except 2MASS J13113560-6245324 and 2MASS J13160379-6242218 (classified as Ofe?YSO and OIf/WN?, 
Photometric and Astrometric Data for the Program Stars

\begin{tabular}{|c|c|c|c|c|c|c|c|c|c|c|c|}
\hline Name & $\begin{array}{c}\alpha(2000) \\
\left({ }^{\circ}\right)\end{array}$ & $\begin{array}{c}\delta(2000) \\
\left(^{\circ}\right)\end{array}$ & $\begin{array}{c}J \\
(\mathrm{mag})\end{array}$ & $\begin{array}{c}H \\
(\mathrm{mag})\end{array}$ & $\begin{array}{c}K_{S} \\
(\mathrm{mag})\end{array}$ & $\begin{array}{c}G \\
(\mathrm{mag})\end{array}$ & $\begin{array}{c}\text { BR } \\
\text { mag }\end{array}$ & $\begin{array}{c}\mathrm{RP} \\
(\mathrm{mag})\end{array}$ & $\begin{array}{c}\pi \\
\text { (mas) }\end{array}$ & $\begin{array}{c}\mu_{\alpha} \cos \delta \\
\text { (mas) }\end{array}$ & $\begin{array}{c}\mu_{\delta} \\
\text { (mas) }\end{array}$ \\
\hline 2M13103971-6243315 & 197.665480 & -62.725430 & 12.570 & 12.310 & 12.420 & $13.985 \pm 0.001$ & $14.323 \pm 0.002$ & $13.456 \pm 0.001$ & $0.4136 \pm 0.0207$ & $-5.2500 \pm 0.0260$ & $-1.5520 \pm 0.0280$ \\
\hline $2 \mathrm{M} 13110621-6256547$ & 197.775880 & -62.948536 & 14.990 & 13.930 & 12.940 & $18.880 \pm 0.007$ & $20.572 \pm 0.107$ & $17.528 \pm 0.023$ & $0.3550 \pm 0.2124$ & $-5.5540 \pm 0.2820$ & $-0.4860 \pm 0.3140$ \\
\hline 2M13112057-6243378 & 197.835720 & -62.727169 & 12.880 & 12.370 & 12.080 & $15.682 \pm 0.001$ & $16.852 \pm 0.004$ & $14.574 \pm 0.002$ & $0.2361 \pm 0.0545$ & $-6.2480 \pm 0.0640$ & $-0.5620 \pm 0.0660$ \\
\hline 2M13112385-6240410 & 197.849400 & -62.678082 & 11.750 & 10.180 & 9.310 & $18.155 \pm 0.007$ & $20.927 \pm 0.126$ & $16.300 \pm 0.025$ & $0.1431 \pm 0.2354$ & $-7.4210 \pm 0.2700$ & $-0.8790 \pm 0.2930$ \\
\hline 2M13113560-6245324 & 197.898340 & -62.759010 & 15.980 & 14.010 & 12.640 & & $\ldots$ & $\ldots$ & & $\ldots$ & $\ldots$ \\
\hline $2 \mathrm{M} 13113936-6233138$ & 197.914005 & -62.553837 & 11.310 & 10.110 & 9.430 & $16.378 \pm 0.001$ & $19.315 \pm 0.048$ & $14.783 \pm 0.007$ & $-0.2045 \pm 0.2245$ & $-7.1120 \pm 0.2630$ & $-1.2680 \pm 0.2680$ \\
\hline 2M13115442-6247078 & 197.976770 & -62.785511 & 12.510 & 10.870 & 9.810 & $18.690 \pm 0.002$ & $20.899 \pm 0.159$ & $16.828 \pm 0.012$ & $0.1750 \pm 0.2655$ & $-7.1190 \pm 0.3390$ & $-0.8240 \pm 0.3540$ \\
\hline 2M13120905-6243267 & 198.037740 & -62.724087 & 10.210 & 8.570 & 7.580 & $16.316 \pm 0.002$ & $\ldots$ & $\ldots$ & $0.0145 \pm 0.1612$ & $-6.9420 \pm 0.2060$ & $-0.9060 \pm 0.2390$ \\
\hline $2 \mathrm{M} 13122130-6240125$ & 198.088780 & -62.670162 & 10.750 & 9.570 & 8.770 & $16.024 \pm 0.008$ & $18.865 \pm 0.028$ & $14.113 \pm 0.008$ & & & $\ldots$ \\
\hline 2M13122546-6244417 & 198.106120 & -62.744923 & 9.810 & 8.480 & 7.650 & $15.064 \pm 0.001$ & $18.232 \pm 0.015$ & $13.467 \pm 0.007$ & $0.2560 \pm 0.1037$ & $-6.7810 \pm 0.1340$ & $-0.1270 \pm 0.1410$ \\
\hline $2 \mathrm{M} 13122653-6243107$ & 198.110556 & -62.719662 & 10.210 & 9.080 & 8.470 & $14.961 \pm 0.002$ & $17.762 \pm 0.016$ & $13.436 \pm 0.009$ & $0.2767 \pm 0.0$ & $-7.1230 \pm 0.1060$ & $-0.3520 \pm 0.1170$ \\
\hline 2M13122855-6241438 & 198.118970 & -62.695522 & 8.260 & 7.270 & 6.610 & $12.613 \pm 0.001$ & $14.859 \pm 0.007$ & $11.177 \pm 0.005$ & $0.2891 \pm 0.0694$ & $-7.1400 \pm 0.0820$ & $-0.6070 \pm 0.1000$ \\
\hline 2M13122965-6234341 & 198.123570 & -62.576149 & 12.990 & 10.510 & 9.540 & $20.755 \pm 0.011$ & $21.130 \pm 0.155$ & $366 \pm 0.058$ & $-2.0311 \pm 1.7502$ & $-8.6670 \pm 2.9370$ & $-1.2980 \pm 2.2980$ \\
\hline 2M13123199-6243583 & 198.133322 & -62.732887 & 11.230 & 10.070 & 9.430 & $16.585 \pm 0.001$ & $19.220 \pm 0.049$ & $14.636 \pm 0.033$ & $-0.1776 \pm 0.1295$ & $-7.1170 \pm 0.1620$ & $-0.0730 \pm 0.1970$ \\
\hline 2M13123629-6239301 & 198.151253 & -62.658379 & 11.680 & 10.640 & 10.170 & $16.379 \pm 0.001$ & $19.212 \pm 0.038$ & $14.836 \pm 0.007$ & $0.0117 \pm 0.0948$ & $-7.0870 \pm 0.1100$ & $-0.3010 \pm 0.1410$ \\
\hline $2 \mathrm{M} 13125612-6243503$ & 198.233839 & -62.730652 & 10.460 & 9.570 & 9.160 & $14.730 \pm 0.004$ & $16.957 \pm 0.007$ & $13.233 \pm 0.004$ & & & $\ldots$ \\
\hline 2M13130268-6242256 & 198.261202 & -62.707127 & 10.790 & 9.810 & 9.330 & $15.383 \pm 0.001$ & $18.093 \pm 0.012$ & $13.876 \pm 0.005$ & $0.1701 \pm 0.0849$ & $-6.8990 \pm 0.0990$ & $-0.2120 \pm 0.1220$ \\
\hline 2M13130841-6239275 & 198.285041 & -62.657639 & 11.420 & 10.270 & 9.750 & $16.260 \pm 0.002$ & $19.194 \pm 0.038$ & $14.704 \pm 0.009$ & $0.1415 \pm 0.0969$ & $-7.2600 \pm 0.1110$ & $-0.5480 \pm 0.1370$ \\
\hline 2M13131324-6245056 & 198.305192 & -62.751556 & 9.840 & 9.060 & 8.720 & $13.745 \pm 0.001$ & $15.729 \pm 0.006$ & $12.382 \pm 0.003$ & $0.1830 \pm 0.0550$ & $-7.1050 \pm 0.0690$ & $-0.4730 \pm 0.0800$ \\
\hline 2M13132400-6245070 & 198.350010 & -62.751968 & 10.630 & 9.930 & 9.620 & $14.249 \pm 0.001$ & $15.906 \pm 0.005$ & $12.921 \pm 0.002$ & $-0.1452 \pm 0.0716$ & $-7.6880 \pm 0.0840$ & $-1.2400 \pm 0.1040$ \\
\hline 2M13134553-6335116 & 198.439746 & -63.586582 & 7.500 & 7.490 & 7.440 & $7.913 \pm 0.001$ & $8.026 \pm 0.001$ & $7.708 \pm 0.001$ & $1.2439 \pm 0.4195$ & $-4.0110 \pm 0.6700$ & $-4.8700 \pm 0.6860$ \\
\hline 2M13144440-6334518 & 198.685003 & -63.581062 & 6.530 & 6.550 & 6.540 & $6.942 \pm 0.000$ & $6.973 \pm 0.007$ & $6.762 \pm 0.008$ & $0.9569 \pm 0.5785$ & $2.9610 \pm 0.9210$ & $-0.9350 \pm 0.9010$ \\
\hline 2M13160379-6242218 & 199.015810 & -62.706081 & 14.080 & 12.020 & 10.540 & $19.659 \pm 0.006$ & $20.776 \pm 0.142$ & $17.975 \pm 0.025$ & $0.5182 \pm 0.4951$ & $-7.0470 \pm 0.8500$ & $-0.0730 \pm 0.5490$ \\
\hline 2M13165955-6247413 & 199.248170 & -62.794830 & 13.030 & 12.720 & 12.430 & $14.498 \pm 0.000$ & $14.937 \pm 0.002$ & $13.874 \pm 0.001$ & $0.3335 \pm 0.0250$ & $-5.1470 \pm 0.0310$ & $0.7730 \pm 0.0320$ \\
\hline 2M13174367-6251410 & 199.431970 & -62.861397 & 11.560 & 10.890 & 10.630 & $15.036 \pm 0.008$ & $15.809 \pm 0.020$ & $13.459 \pm 0.012$ & $0.8481 \pm 0.4632$ & $-5.9230 \pm 0.5210$ & $0.4500 \pm 0.5810$ \\
\hline 2M13181288-6239067 & 199.553700 & -62.651882 & 12.950 & 12.070 & 11.300 & $15.535 \pm 0.001$ & $16.391 \pm 0.004$ & $14.570 \pm 0.003$ & $0.4215 \pm 0.0431$ & $-4.4230 \pm 0.0450$ & $-2.1250 \pm 0.0500$ \\
\hline 2M13183535-6229284 & 199.647326 & -62.491245 & 7.470 & 7.460 & 7.440 & $7.911 \pm 0.000$ & $8.037 \pm 0.001$ & $7.695 \pm 0.001$ & $0.4413 \pm 0.0472$ & $-4.1810 \pm 0.0510$ & $-2.1740 \pm 0.0540$ \\
\hline 2M13190396-6234102 & 199.766510 & -62.569504 & 10.020 & 9.920 & 9.800 & $10.677 \pm 0.001$ & $10.839 \pm 0.002$ & $10.386 \pm 0.002$ & $0.3457 \pm 0.0370$ & $-4.3010 \pm 0.0430$ & $-1.8200 \pm 0.0430$ \\
\hline 2M13195292-6240096 & 199.970540 & -62.669346 & 13.170 & 12.800 & 12.580 & $15.353 \pm 0.001$ & $16.021 \pm 0.004$ & $14.494 \pm 0.002$ & $0.2789 \pm 0.0425$ & $-4.7370 \pm 0.0540$ & $-1.9940 \pm 0.0530$ \\
\hline
\end{tabular}


Table 3

Basic Parameters of the Program Stars

\begin{tabular}{|c|c|c|c|c|c|c|c|}
\hline Name & $\mathrm{S} / \mathrm{N}$ & Type & $\begin{array}{c}\mathrm{RV} \\
\left(\mathrm{km} \mathrm{s}^{-1}\right)\end{array}$ & $\begin{array}{l}\mathrm{Dis}_{\text {sp }} \\
\text { (pc) }\end{array}$ & $\begin{array}{l}\text { Dis }_{\text {Rest }} \\
\text { (pc) }\end{array}$ & $\begin{array}{c}E(\mathrm{BP}-\mathrm{RP}) \\
(\mathrm{mag})\end{array}$ & $\begin{array}{c}E(J-K) \\
(\mathrm{mag})\end{array}$ \\
\hline 2M13103971-6243315 & 26 & B3-4V & $-56.21 \pm 6.94$ & 5480 & $2262 \pm 151$ & $\cdots$ & 0.25 \\
\hline 2M13110621-6256547 & 10 & B2-3V & $-34.75 \pm 7.16$ & 4930 & $2749 \pm 2331$ & $\ldots$ & 2.16 \\
\hline 2M13112057-6243378 & 30 & B5-6V & $-31.39 \pm 6.31$ & 2880 & $3694 \pm 1136$ & 1.0218 & 0.88 \\
\hline 2M13112385-6240410 & 122 & B0-1V & $-31.08 \pm 6.15$ & 1500 & $3662 \pm 2803$ & $\ldots$ & 2.59 \\
\hline 2M13113560-6245324 & 7 & Ofe?YSO ${ }^{5}$ & $-33.69 \pm 9.03$ & $\cdots$ & $\ldots$ & $\cdots$ & $\cdots$ \\
\hline 2M13113936-6233138 & 103 & O9-B0 & $-45.22 \pm 6.09$ & 2230 & $5042 \pm 3200$ & $\cdots$ & 2.05 \\
\hline 2M13115442-6247078 & 77 & O8-9? & $-34.41 \pm 6.24$ & 2700 & $3465 \pm 2808$ & $\cdots$ & 2.87 \\
\hline 2M13120905-6243267 & 255 & WN8-9 ${ }^{1}$ & $-41.12 \pm 7.14$ & $\ldots$ & $4843 \pm 3015$ & $\ldots$ & $\ldots$ \\
\hline 2M13122130-6240125 & 153 & $\mathrm{WC}^{2}$ & $-38.71 \pm 8.29$ & $\ldots$ & $3631 \pm 2708$ & $\ldots$ & 2.05 \\
\hline 2M13122546-6244417 & 256 & $\mathrm{WN}^{1}$ & $-41.27 \pm 8.79$ & $\cdots$ & $3380 \pm 1807$ & $\cdots$ & 1.95 \\
\hline 2M13122653-6243107 & 171 & O6-7I & $-41.23 \pm 5.68$ & 2680 & $3221 \pm 1448$ & 1.4298 & 1.95 \\
\hline 2M13122855-6241438 & 150 & $\mathrm{WNLh}^{1} / \mathrm{O} 7-8 \mathrm{I}$ & $-48.18 \pm 5.90$ & $\cdots$ & $3124 \pm 1067$ & 1.0985 & 1.95 \\
\hline 2M13122965-6234341 & 79 & O8-9V & $-38.58 \pm 5.77$ & 1590 & $3244 \pm 3113$ & $\cdots$ & 3.66 \\
\hline 2M13123199-6243583 & 88 & O6-7V & $-40.08 \pm 5.92$ & 2960 & $6774 \pm 3512$ & $\cdots$ & 1.98 \\
\hline 2M13123629-6239301 & 79 & O5-6V & $-43.55 \pm 6.17$ & 5080 & $6150 \pm 3115$ & 0.641 & 1.72 \\
\hline 2M13125612-6243503 & 137 & $05-6 \mathrm{~V}$ & $-43.55 \pm 6.37$ & 3360 & $\cdots$ & $\cdots$ & 1.51 \\
\hline 2M13130268-6242256 & 139 & O7-8V & $-43.64 \pm 5.72$ & 2690 & $4356 \pm 2165$ & $\cdots$ & 1.67 \\
\hline 2M13130841-6239275 & 116 & $07-8 V^{3}$ & $-43.40 \pm 5.66$ & 3090 & $4570 \pm 2439$ & $\ldots$ & 1.89 \\
\hline 2M13131324-6245056 & 170 & O4-5I & $-43.50 \pm 5.00$ & 4370 & $4432 \pm 1577$ & 1.0682 & 1.32 \\
\hline 2M13132400-6245070 & 108 & O6-7V & $-42.58 \pm 5.97$ & 3880 & $9559 \pm 3913$ & $\ldots$ & 1.23 \\
\hline 2M13134553-6335116 & 91 & O9-B0V & $-40.12 \pm 5.77$ & 1230 & $964 \pm 1490$ & 0.553 & 0.27 \\
\hline 2M13144440-6334518 & 87 & O9-B0V & $-39.26 \pm 5.67$ & 820 & $1998 \pm 2816$ & $\cdots$ & 0.20 \\
\hline 2M13160379-6242218 & 31 & OIf*/WN? & $-44.93 \pm 8.03$ & & $2556 \pm 2767$ & $\cdots$ & 0.68 \\
\hline 2M13165955-6247413 & 18 & B4-5V & $-45.01 \pm 6.23$ & 4170 & $2760 \pm 276$ & $\cdots$ & 0.68 \\
\hline 2M13174367-6251410 & 40 & B1-2V & $-45.70 \pm 6.09$ & 2920 & $1748 \pm 2399$ & $\cdots$ & 1.06 \\
\hline 2M13181288-6239067 & 35 & B2-3V & $-45.70 \pm 6.15$ & 2550 & $2229 \pm 317$ & $\cdots$ & 1.76 \\
\hline 2M13183535-6229284 & 72 & O6-7V $\mathrm{V}^{4}$ & $-47.38 \pm 5.89$ & 1810 & $2137 \pm 319$ & 0.3675 & 0.24 \\
\hline 2M13190396-6234102 & 57 & O9-B0 & $-46.71 \pm 5.72$ & 3510 & $2672 \pm 390$ & 0.6453 & 0.43 \\
\hline 2M13195292-6240096 & 109 & B1-2V & $-45.70 \pm 6.09$ & 7790 & $3233 \pm 660$ & $\ldots$ & 0.71 \\
\hline
\end{tabular}

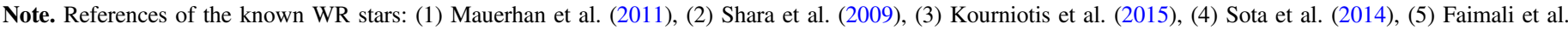
(2012).

respectively) are B-type main-sequence stars. The spectrum of 2MASS J13165540-6242318 is not shown and excluded from the analysis, because of some instrument defects in the fiber producing a low $\mathrm{S} / \mathrm{N}=5$.

To study the three-dimensional placement of variable stars in the space, we used the $X Y Z$ coordinate system. The $X Y Z$ coordinates are calculated from a conversion of spherical Galactic coordinates to Cartesian coordinates, using the Gaia distance estimates (Table 3, column 5, rest distance). Figure 11 shows the $X Z$ and $Y Z$ planes. Although the errors are big due to uncertain distance estimates, the $|z|$ distribution is clearly concentrated very close to the galactic plane, and the variable stars follow the same trend.

Table 2 summarizes the photometric and astrometric data of the program stars. The magnitudes are taken from VVV and Gaia DR2 databases.

Table 3 summarizes the derived basic parameters data of the program stars. Dist ${ }_{\mathrm{sp}}$ is a spectroscopically calculated distance, while Dist ${ }_{\text {Rest }}$ is the distance taken from the Bailer-Jones et al. (2018) catalog.

\section{Discussion}

As noted in the introduction, the massive stellar content of the G 305 star-forming region was previously investigated by Clark \& Porter (2004), Leistra et al. (2005), Mauerhan et al. (2011), Chené et al. (2012), Davies et al. (2012), and Zhekov et al. (2014). According to SIMBAD, within a radius of 1.7
311 stars are classified as WR, O, B, and OB stars; 232 of them (including our sample) have Gaia DR2 measurements. Figure 12 shows their proper motion and distance distributions. As expected, the B stars are the biggest group (54\%), followed by $\mathrm{O}(16 \%)$ and WR stars $(7 \%)$. But at least $23 \%$ of this Simbad sample might contain dubious spectral classifications (such as $\mathrm{OB}, \mathrm{OB}+$ or $\mathrm{OB}-$ ), thus this distribution should be taken with caution.

The proper motion diagrams show clear concentration of WR and $O$ stars toward Danks 1 and 2 massive young clusters, confirming the hypothesis of recent bursts of star formation in this part of G 305. In a subsequent paper we will discuss the membership of these stars, as well as search for runway stars. The B stars also indicate some concentration toward these clusters, but they are much more uniformly distributed. The Gaia DR2 mean distances (Bailer-Jones et al. 2018) calculated from the WR and $\mathrm{O}$ star samples are in very good agreement, with $\mathrm{WR}_{\text {dist }}=3.76 \pm 1.26 \mathrm{kpc}$ and $\mathrm{O}_{\text {dist }}=3.63 \pm 1.76 \mathrm{kpc}$, respectively.

Since early works based on Einstein X-ray telescope data, it has been known that O-type stars emit X-rays (see Nebot Gómez-Morán \& Oskinova 2018 and references therein for more details). In addition to the binary hypothesis, X-ray emission from $\mathrm{O}$ stars is attributed to the presence of plasma heated by shocks intrinsic to radiatively driven stellar winds (Feldmeier et al. 1997). An alternative explanation, involving stellar magnetism (Waldron \& Cassinelli 2007), is also 

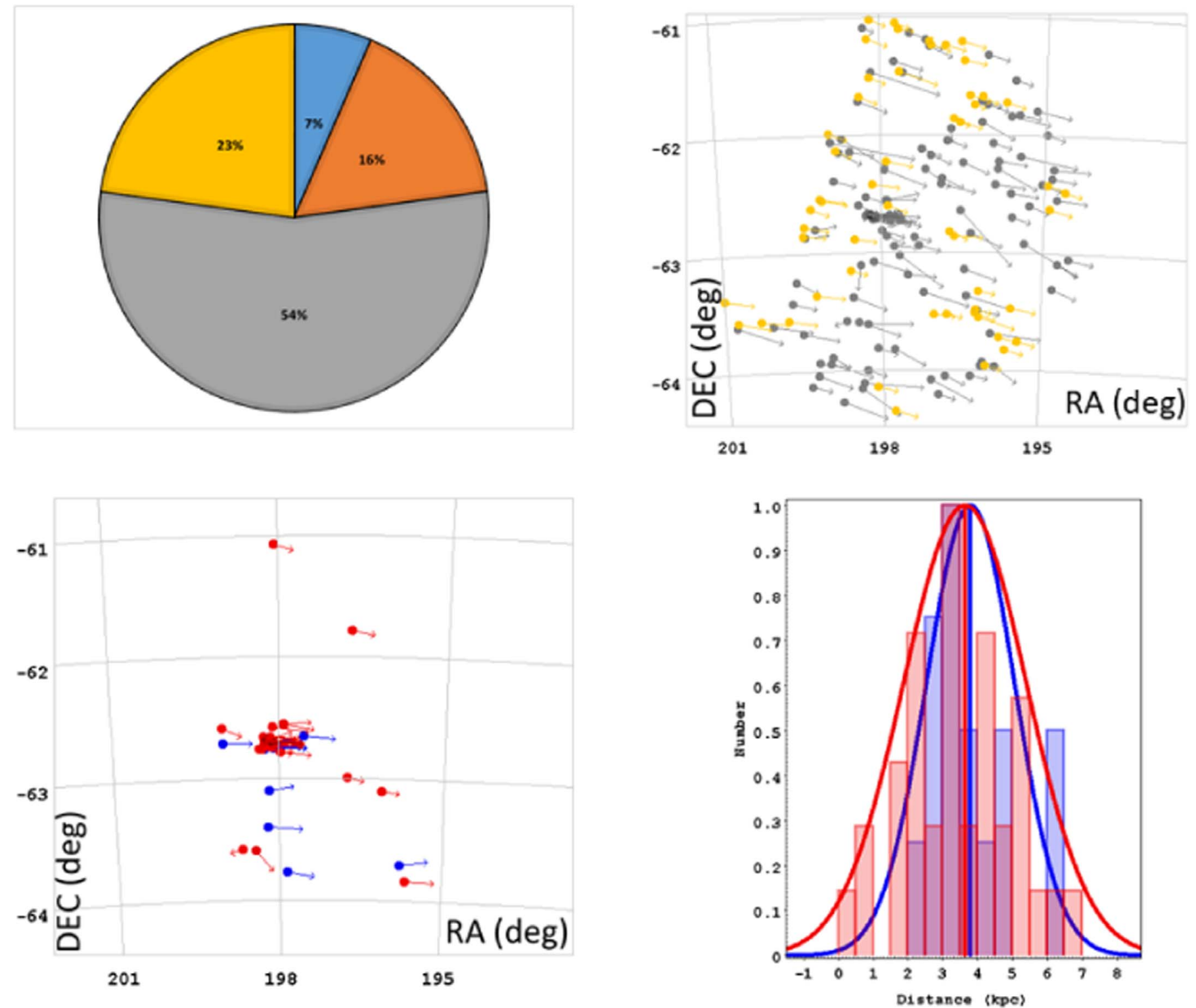

Figure 12. Top left: histogram distribution of relative frequency of WR (blue), O (red), B (gray), and unclassified OB stars (yellow) with respect to the whole sample. Bottom left: proper motion distribution of WR and $\mathrm{O}$ stars, scaled by a factor of 2 for visibility. Top right: proper motion distribution of $\mathrm{B}$ and OB stars, scaled by a factor of 2 for visibility. Bottom right: histogram distribution of distances of WR and O stars. The solid lines draw the Gaussian distributions.

considered; in this case the X-ray-emitting plasma could be associated with stellar spots. A series of papers (for example Nebot Gómez-Morán \& Oskinova 2018) have found that X-ray luminosities of dwarf and giant $\mathrm{O}$ stars correlate with their bolometric luminosity and wind parameters. In our case, from 28 observed OB candidates with X-ray emission, we confirmed $54 \%$ of them. Only five stars show a typical spectrum for red stars. The remaining stars have $\mathrm{S} / \mathrm{Ns}$ that are too poor to classify them property. The sample is too small to search for the previously mentioned correlation, but it confirms that X-rays can be successfully used to identify new OB candidates.

Binary systems are well known to produce X-ray emission, and the sample reported here contains four known binary systems from literature. We identify two new binary system candidates: 2MASS J13112057-6243378 (on the basis of its APOGEE spectrum) and 2MASS J13160379-6242218 (based on its $K_{s}$ variability).
We believe that this investigation serves as a prologue to the next generation of telescopes and instruments, such as the James Webb Space Telescope. It is expected to measure the abundances, composition, and production rates of various dusty sources, including mass loss and evolved stars (see, for example, the approved projects "Decoding Smoke Signals in the Glare of a WR Binary" with PI R. Lau; and "Radiative Feedback from Massive Stars as Traced by Multiband Imaging and Spectroscopic Mosaics" with PI O. Berne). Thus, using the current surveys in the near-IR (such as VVV and APOGEE), we can prepare a list of interesting objects in star-forming regions in our Galaxy for further and deeper follow-up.

We acknowledge a very constructive report by the anonymous referee. Funding for the Sloan Digital Sky Survey IV has been provided by the Alfred P. Sloan Foundation, the U.S. Department of Energy Office of Science, and the Participating Institutions. SDSS-IV acknowledges support and 
resources from the Center for High-Performance Computing at the University of Utah. The SDSS website is www.sdss.org. SDSS-IV is managed by the Astrophysical Research Consortium for the Participating Institutions of the SDSS Collaboration including the Brazilian Participation Group, the Carnegie Institution for Science, Carnegie Mellon University, the Chilean Participation Group, the French Participation Group, Harvard-Smithsonian Center for Astrophysics, Instituto de Astrofísica de Canarias, The Johns Hopkins University, Kavli Institute for the Physics and Mathematics of the Universe (IPMU)/University of Tokyo, the Korean Participation Group, Lawrence Berkeley National Laboratory, Leibniz Institut für Astrophysik Potsdam (AIP), Max-Planck-Institut für Astronomie (MPIA Heidelberg), Max-Planck-Institut für Astrophysik (MPA Garching), Max-Planck-Institut für Extraterrestrische Physik (MPE), National Astronomical Observatories of China, New Mexico State University, New York University, University of Notre Dame, Observatário Nacional/MCTI, The Ohio State University, Pennsylvania State University, Shanghai Astronomical Observatory, United Kingdom Participation Group, Universidad Nacional Autónoma de México, University of Arizona, University of Colorado Boulder, University of Oxford, University of Portsmouth, University of Utah, University of Virginia, University of Washington, University of Wisconsin, Vanderbilt University, and Yale University. We gratefully acknowledge data from the ESO Public Survey program ID 179.B-2002 taken with the VISTA telescope, and products from the Cambridge Astronomical Survey Unit (CASU). This work has made use of data from the European Space Agency (ESA) mission Gaia (https://www.cosmos.esa. int/gaia), processed by the Gaia Data Processing and Analysis Consortium (DPAC, https://www.cosmos.esa.int/web/gaia/ dpac/consortium). Funding for the DPAC has been provided by national institutions, in particular the institutions participating in the Gaia Multilateral Agreement. Support is provided by the Ministry for the Economy, Development and Tourism, Programa Iniciativa Cientica Milenio grant IC120009, awarded to the Millennium Institute of Astrophysics (MAS). D.M. is supported by the BASAL Center for Astrophysics and Associated Technologies (CATA) through grant AFB170002 and by FONDECYT No. 1170121. C.R.Z. acknowledges support from program UNAM-DGAP- PAPIIT IN 108117, Mexico. S.R.A. thanks the support by the FONDECYT Iniciacion project No. 11171025 and the CONICYT + PAI "Concurso Nacional Insercion de Capital Humano Avanzado en la Academia 2017" project PAI 79170089. A. Roman-Lopes acknowledges financial support provided in Chile by Comisión Nacional de Investigación Científica y Tecnológica (CONICYT) through the FONDECYT project 1170476 and by the QUIMAL project 130001.

\section{ORCID iDs}

Jura Borissova (ํ) https://orcid.org/0000-0002-5936-7718 Alexandre Roman-Lopes (i) https://orcid.org/0000-00021379-4204

Kevin Covey @il https://orcid.org/0000-0001-6914-7797
Nicolas Medina (1) https://orcid.org/0000-0002-4684-1927

Carlos Roman-Zuniga (i) https://orcid.org/0000-00018600-4798

M. A. Kuhn (ㄷ) https://orcid.org/0000-0002-0631-7514

Philip Lucas (1) https://orcid.org/0000-0002-8872-4462

Sebastian Ramirez Alegria (i) https://orcid.org/0000-00027754-9785

Dante Minniti 자 https://orcid.org/0000-0002-7064-099X

Marina Kounkel (i) https://orcid.org/0000-0002-5365-1267

Guy Stringfellow (i) https://orcid.org/0000-0003-1479-3059

Genaro Suárez iㅏ https://orcid.org/0000-0002-2011-4924

\section{References}

Bailer-Jones, C. A. L., Rybizki, J., Fouesneau, M., Mantelet, G., \& Andrae, R. 2018, AJ, 156, 58

Borissova, J., Bonatto, C., Kurtev, R., et al. 2011, A\&A, 532, A131

Borissova, J., Chené, A.-N., Ramírez Alegría, S., et al. 2014, A\&A, 569, 24

Borissova, J., Ramírez Alegría, S., Alonso, J., et al. 2016, AJ, 152, 74

Bressan, A., Marigo, P., Girardi, L., et al. 2012, MNRAS, 427, 127

Chené, A.-N., Borissova, J., Clarke, J. R. A., et al. 2012, A\&A, 545, A54

Clark, J. S., Davies, B., \& Thompson, M. A. 2011, BSRSL, 80, 223

Clark, J. S., \& Porter, J. M. 2004, A\&A, 427, 839

Coşkunoğlu, B., Ak, S., Bilir, S., et al. 2011, MNRAS, 412, 1237

Contreras Peña, C., Lucas, P. W., Minniti, D., et al. 2017, MNRAS, 465, 3011

Davies, B., Clark, J. S., Trombley, C., et al. 2012, MNRAS, 419, 1871

Evans, I. N., Primini, F. A., Glotfelty, K. J., et al. 2010, ApJS, 189, 37

Faimali, A., Thompson, M. A., Hindson, L., et al. 2012, MNRAS, 426, 402

Feldmeier, A., Puls, J., \& Pauldrach, A. W. A. 1997, A\&A, 322, 878

Gaia Collaboration, Brown, A. G. A., Vallenari, A., et al. 2018a, A\&A, 616, A1

Gaia Collaboration, Helmi, A., van Leeuwen, F., et al. 2018b, A\&A, 616, A12

Gaia Collaboration, Prusti, T., de Bruijne, J. H. J., et al. 2016, A\&A, 595, A1

Hindson, L., Thompson, M. A., Urquhart, J. S., et al. 2012, MNRAS, 421,3418

Hindson, L., Thompson, M. A., Urquhart, J. S., et al. 2013, MNRAS, 435, 2003

Hindson, L., Thompson, M. A., Urquhart, J. S., Clark, J. S., \& Davies, B. 2010, MNRAS, 408, 1438

Hindson, L. P. 2012, PhD thesis, Univ. Hertfordshire

Kourniotis, M., Bonanos, A. Z., Williams, S. J., et al. 2015, A\&A, 582, A42

Leistra, A., Cotera, A. S., Liebert, J., \& Burton, M. 2005, AJ, 130, 1719

Lindegren, L., Hernandez, J., Bombrun, A., et al. 2018, A\&A, 616, A2

Martins, F., \& Plez, B. 2006, A\&A, 457, 637

Marton, G., Tóth, L. V., Paladini, R., et al. 2016, MNRAS, 458, 3479

Mauerhan, J. C., Van Dyk, S. D., \& Morris, P. W. 2011, AJ, 142, 40

Medina, N., Borissova, J., Bayo, A., et al. 2018, ApJ, 864, 11

Minniti, D., Lucas, P. W., Emerson, J. P., et al. 2010, NewA, 15, 433

Nebot Gómez-Morán, A., \& Oskinova, L. M. 2018, A\&A, 620, A89

Nidever, D. L., Holtzman, J. A., Allende Prieto, C., et al. 2015, AJ, 150, 173

Robitaille, T. P., Meade, M. R., Babler, B. L., et al. 2008, AJ, 136, 2413

Roman-Lopes, A., Román-Zúñiga, C., Tapia, M., et al. 2018, ApJ, 855, 68

Saito, R. K., Hempel, M., Minniti, D., et al. 2012, A\&A, 537, 107

Shara, M. M., Moffat, A. F. J., Gerke, J., et al. 2009, AJ, 138, 402

Skrutskie, M. F., Cutri, R. M., Stiening, R., et al. 2006, AJ, 131, 1163

Sota, A., Maíz Apellániz, J., Morrell, N. I., et al. 2014, ApJS, 211, 10

Straižys, V., \& Lazauskaitè, R. 2009, BaltA, 18, 19

Urquhart, J. S., Figura, C. C., Moore, T. J. T., et al. 2014, MNRAS, 437, 1791

Vázquez, R. A., Baume, G. L., Feinstein, C., Nuñez, J. A., \& Vergne, M. M. 2005, A\&A, 430, 471

Waldron, W. L., \& Cassinelli, J. P. 2007, ApJ, 668, 456

Williams, P. M., van der Hucht, K. A., van Wyk, F., et al. 2012, MNRAS, 420, 2526

Zhekov, S. A., Tomov, T., Gawronski, M. P., et al. 2014, MNRAS, 445, 1663 